\title{
Discrete-time feedback stabilization
}

\author{
WOJCIECH MITKOWSKI, WALDEMAR BAUER and MARTA ZAGÓROWSKA
}

\begin{abstract}
This paper presents an algorithm for designing dynamic compensator for infinitedimensional systems with bounded input and bounded output operators using finite dimensional approximation. The proposed method was then implemented in order to find the control function for thin rod heating process. The optimal sampling time was found depending on discrete output measurements.
\end{abstract}

Key words: stabilization, feedback stabilization, finite dimensional stabilization, infinite dimensional systems, finite dimensional approximations, continuous-discrete system.

\section{Introduction}

One of the main areas of automatic control is related to stabilization problems. Usually, in real time application, an algorithm consisting of two stages is used: 1. Bring the system to the valid region of linearization. 2. Stabilize the system using linear approximation. This approach is justified by topological similarity of a nonlinear system and its linearization (valid only for hyperbolic systems without purely imaginary eigenvalues).

Feedback design (design of the stabilizing controller) depends on the system form (usually we have either differential equations or transfer function for time invariant systems).

The design of finite dimensional feedback is useful due to multiple reasons: 1 . It is possible to use simple, finite-dimensional methods, e.g., Lyapunov functions and in consequence, Lyapunov equations strictly linked with algebraic Riccati equations. 2. Some of the systems have predefined structure, e.g., the hoisting machine (long line is a distributed system, and the drive may be modeled with finite-dimensional system).

The design of finite-dimensional controllers for infinite systems with finite set of unstable modes (or at least weakly damped ones) is widely analyzed in literature. This class of the systems was described by Triggiani (1975) [34], or even earlier by Fattorini (1967) [11]. Using small disturbance methods and building appropriate invariant sets, Schumacher $(1981,1983)$ [30, 31] proposed finite dimensional stabilizing controllers

The Authors are with AHG University of Science and Technology, Krakow, Poland. W. Mitkowski is the corresponding author, e-mail: wojciech.mitkowski@agh.edu.pl. W. Mitkowski is the corresponding author.

This work was supported by the AGH (Poland) - the project No 11.11.120.817.

Received 14.12.2016. Revised 30.04.2017. 
for distributed and delayed systems (see also Kamen (1985) [14]). Similar results were obtained by Curtain (1984) [4] for parabolic systems with infinite input-output operators. Also the works of Curtain and Salomon (1986) [5], and Sakawa (1983, 1984, 1985) [27-29] are worth noticing. Balas (1983) [2] proposed a finite dimensional dynamic compensator for finite dimensional approximations of infinite systems. Similar methods were proposed by Kobayashi (1983) [16]. Gibson (1981) [13] used finite dimensional approximation of algebraic Riccati equation. The detailed description of those works was done, e.g., by Mitkowski (1991) [20] with 229 books and papers analyzed.

The design of stabilizing controllers is still an interesting problem (see, e.g. Przyşuski (2014) [26]), especially as there are more efficient numerical tools. Thanks to computers, nowadays, we can analyze complex mathematical models of distributed parameter systems, e.g. models of non-integer order Obrźczka (2014) [22], Sierociuk (2015) [32], Oprzêdkiewicz (2016) [24] which sometimes better describe real systems.

In this work, we focused on an algorithm of stabilization of linear infinite dimensional system with bounded input and bounded output operators and with finite set of unstable modes (weakly damped) using finite discrete stabilization. As an example, we used diffusion equation which models the heating process of a thin rod.

\section{Problem description}

Consider a closed-loop system (with continuous time) shown in Fig. 1.

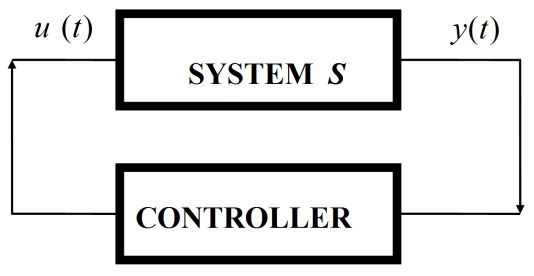

Figure 1: Closed-loop system.

Finite dimensional stabilization problem: for a given infinite system $S$ find a stabilizing controller (finite dimensional) such that the closed-loop system is exponentially stable with predefined damping coefficient.

In digital control, it is necessary to use a discrete system (computer or other device with discrete time). In order to use a discrete stabilizing controller in continuous time system, we need to use the system (see Fig. 2) in the form of a series of pulser, continuous linear system $S$, and ZOH (Zero Order Hold) with input $u(k)$ and output $y(k)$, $k=1,2,3, \ldots$.

If the pulser and $\mathrm{ZOH}$ work synchronously with time step $h>0$, then the parameters of discrete linear system $S^{d}$ denoted for simplicity with $A, B, C$ are given by the formulas 


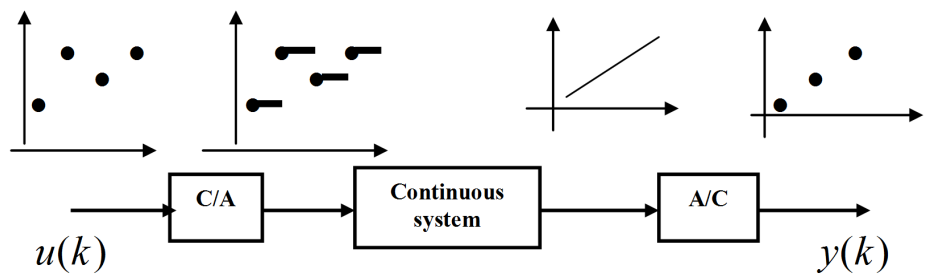

Figure 2: Continuous-discrete system.

calculated on the basis of continuous system:

$$
A:=e^{A h}, \quad B:=\int_{0}^{h} e^{A t} B d t, \quad C:=C
$$

For a valid controller (both continuous and discrete), we need the controllability and observability of continuous system $S$. The conditions for time step $h>0$ which guarantee that the discrete system is also controllable and observable are known and may be found, e.g., in Mitkowski (1991, p. 141) [20].

\section{The decomposition of the system}

There is a group of infinite dimensional systems which can be stabilized using finite dimensional methods. Let us now consider a system (see for example Pazy (1983) [25], Slemrod (1974) [33], Wang (1972) [35], Curtain and Pritchard (1978) [7], Curtain and Zwart (1995) [8])

$$
\begin{array}{llrl}
\dot{x}(t) & =A x(t)+B u(t), & & y(t)=C x(t) \\
x(t) & \in X, \quad u(t) \in U, & & y(t) \in Y
\end{array}
$$

For further use we will denote it as $S(A, B, C)$. Let us now assume that (2) fulfills the following conditions:

- $X, Y, U-$ Hilbert spaces, $\operatorname{dim} U<+\infty$.

- $A$ is an infinitesimal generator $C_{0}$ of semi-group $T_{A}(t)$, for $t \geqslant 0$ in $X$.

- $B \in L(U, X), C \in L(X, Y)$ are bounded.

- $A$ is a discrete operator with finite number of eigenvalues with $\operatorname{Re} s>\beta, \beta<+\infty$. 
Taking into account the conditions above, we can decompose (2) into (Triggiani (1975) [34]):

$$
\begin{gathered}
{\left[\begin{array}{c}
\dot{x}_{1}(t) \\
\dot{x}_{2}(t) \\
\dot{x}_{3}(t)
\end{array}\right]=\left[\begin{array}{ccc}
A_{1} & 0 & 0 \\
0 & A_{2} & 0 \\
0 & 0 & A_{3}
\end{array}\right]\left[\begin{array}{l}
x_{1}(t) \\
x_{2}(t) \\
x_{3}(t)
\end{array}\right]+\left[\begin{array}{l}
B_{1} \\
B_{2} \\
B_{3}
\end{array}\right] u(t),} \\
y(t)=C_{1} x_{1}(t)+C_{2} x_{2}(t)+C_{3} x_{3}(t), \\
x_{i}(t) \in X_{i}, i=1,2,3, X=X_{1}+X_{2}+X_{3}, \\
\operatorname{dim} X_{1}<+\infty, \quad \operatorname{dim} X_{2}=p<+\infty .
\end{gathered}
$$

The spectrum of $A$ (see (2)) is depicted in the Fig. 3. The operator $A_{1}$ is responsible for unstable (or weakly damped) part of the system (3). The operators $A_{2}$ and $A_{3}$ are exponentially stable.

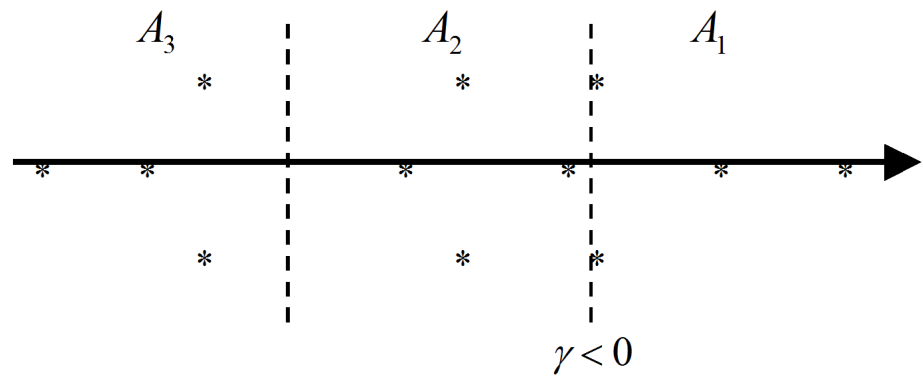

Figure 3: Discrete spectrum of $A$.

Let us now add the following assumptions:

- $\sup \left\{\operatorname{Re} s: s \in \lambda\left(A_{3}\right)<0, \sup \left\{\operatorname{Re} s: s \in \lambda\left(A_{2}\right)\right\}=\gamma<0\right.$.

- The pair $\left(A_{1}, B_{1}\right)$ is controllable, the pair $\left(C_{1}, A_{1}\right)$ is observable.

- $\operatorname{dim} X_{2}=p \rightarrow+\infty \Rightarrow\left\|B_{3}\right\| \rightarrow 0$ and $\left\|C_{3}\right\| \rightarrow 0$.

The last assumption is fulfilled if, e.g., self-adjoint generator $A$ has compact resolvent (the eigenvectors form a basis of the given space). 


\section{Finite-dimensional stabilizing controller}

Let us now consider dynamic feedback Mitkowski (1988 [19, p. 519], 1991, [20, p. 233]) of form:

$$
\begin{aligned}
& {\left[\begin{array}{c}
\dot{w}_{1}(t) \\
\dot{w}_{2}(t)
\end{array}\right]=\left[\begin{array}{cc}
A_{1}-G_{1} C_{1}+B_{1} K_{1} & -G_{1} C_{2} \\
B_{2} K_{1} & A_{2}
\end{array}\right]\left[\begin{array}{c}
w_{1}(t) \\
w_{2}(t)
\end{array}\right]+\left[\begin{array}{c}
G_{1} \\
0
\end{array}\right] y(t),} \\
& u(t)=K_{1} w_{1}(t), \quad w_{i}(t) \in X_{i}, \quad i=1,2 .
\end{aligned}
$$

Let us assume that the conditions mentioned in previous section are fulfilled. There exists a finite dimensional stabilizing controller (5), such that the closed-loop system (2) with (5) is exponentially stable with predefined damping coefficient $\alpha \in(\gamma, 0)$, see Sakawa (1983) [27]. See also Mitkowski (1982, 1986, 1988) [17, 19], Mitkowski (1991, [20, p. 230]) for further details.

The design of feedback (5) may be reduced to finding the matrices $K_{1}$ and $G_{1}$ which can be done using methods known from finite dimensional system's analysis, e.g., LQ design. The desired damping coefficient $\alpha \in(\gamma, 0)$ can be found by increasing $p=$ $\operatorname{dim} X_{2}$.

A discrete version of the controller (Mitkowski (1991, [20, p. 236]) can be obtained using formulas (1) and remembering that the system is asymptotically stable if the eigenvalues lie inside the unit circle. The matrices $K_{1}$ i $G_{1}$ should be found in a way that guarantees that the eigenvalues of matrices $A_{1}+B_{1} K_{1}$ and $A_{1}-G_{1} C_{1}$ lie inside the unit circle (for example, we can set them as zeros).

\section{Example}

Let us now consider the process of heating a thin rod (Oprzedkiewicz $(2003,2016)$ $[23,24])$ depicted in the Fig. 4.

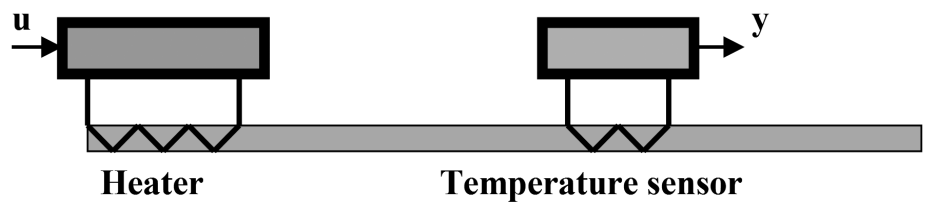

Figure 4: Heating of a thin rod. 
A simplified mathematical model of the analyzed process has the form

$$
\begin{aligned}
& \frac{\partial x(z, t)}{\partial t}=a \frac{\partial^{2} x(z, t)}{\partial z^{2}}-R_{a} x(z, t)+b(z) u(t), \quad t \geqslant 0, \quad z \in[0,1] \\
& \left.\frac{\partial x(z, t)}{\partial z}\right|_{z=0}=\left.\frac{\partial x(z, t)}{\partial z}\right|_{z=1}=0, \quad t \geqslant 0, \\
& x(z, 0)=0, \quad z \in(0,1), \\
& y(t)=\int_{0}^{1} c(z) x(z, t) d z .
\end{aligned}
$$

where

$$
\begin{gathered}
b(z)=\left\{\begin{array}{lll}
1 & \text { for } & 0 \leqslant z \leqslant z_{0} \\
0 & \text { for } & z_{0}<z \leqslant 1
\end{array}\right. \\
c(z)=\left\{\begin{array}{lll}
\bar{c} & \text { for } & z_{1} \leqslant z \leqslant z_{2} \\
0 & \text { for } & 0 \leqslant z<z_{1}
\end{array} \text { and } z_{2}<z \leqslant 1\right. \\
x(z, t)=\sum_{i=0}^{\infty} x_{i}(t) h_{i}(z)
\end{gathered}
$$

After the decomposition, we have system $S(A, B, C, D)$, where

$$
\begin{array}{lll}
A=\operatorname{diag}\left(\lambda_{0}, \lambda_{1}, \lambda_{2}, \ldots \ldots\right), & B=\left[b_{0} b_{1} b_{2} \ldots \ldots \ldots . .\right]^{T}, \\
C=\left[c_{0} c_{1} c_{2} \ldots \ldots \ldots . .\right. & D=0, &
\end{array}
$$

and

$$
\begin{gathered}
X=L^{2}(0,1 ; R), \quad \lambda_{i}=-i^{2} \pi^{2} a-R_{a}, \quad i=0,1,2, \ldots \\
h_{i}(z)=\left\{\begin{array}{c}
1 \quad \text { for } \quad i=0 \\
\sqrt{2} \cos (i \pi z) \quad \text { for } \quad i=1,2,3, \ldots .
\end{array}\right. \\
b_{i}=\int_{0}^{1} b(z) h_{i}(z) d z, \quad c_{i}=\int_{0}^{1} c(z) h_{i}(z) d z,
\end{gathered}
$$

we have the following parameters for model (6) (verified in a laboratory, Oprzedkiewicz $(2003,2004)[26,21])$ :

$$
a=0.000945, R_{a}=0.0271, \bar{c}=25.7922 z_{0}=1 / 13, z_{1}=25 / 52, z_{2}=27 / 52
$$

From (7), we have

$$
\begin{aligned}
& A=\operatorname{diag}(-0.0269-0.0358-0.0624-0.1068-0.1690-0.2490-0.3467 \\
& -0.4621-0.5954-0.7464-0.9152-1.1017-1.3060-1.5281-1.7679 \\
& -2.0255-2.3009-2.5940-2.9049-3.2335-3.5800-3.9441-4.3261 \\
& -4.7258-5.1433)
\end{aligned}
$$




$$
\begin{aligned}
& B=[0.07690 .10770 .10460 .09950 .09260 .08420 .07450 .0638 \\
& 0.05260 .04120 .02990 .01900 .0090-0.0000-0.0077-0.0139 \\
& -0.0187-0.0218-0.0234-0.0235-0.0223-0.0200-0.0168-0.0130 \\
& \quad-0.0087]^{\mathrm{T}} \\
& C=[1.01710-1.4348-0.00001 .4244-0.0000-1.40700 .0000 \\
& 1.3830-0.0000-1.3524-0.00001 .3156-0.0000-1.2729-0.0000 \\
& 1.2246-0.0000-1.1711-0.00001 .1130-0.0000-1.05070 .00000 .9848]
\end{aligned}
$$

In order to perform the simulation, the heating process was implemented with use of Matlab/Simulink environment (see Fig. 5).

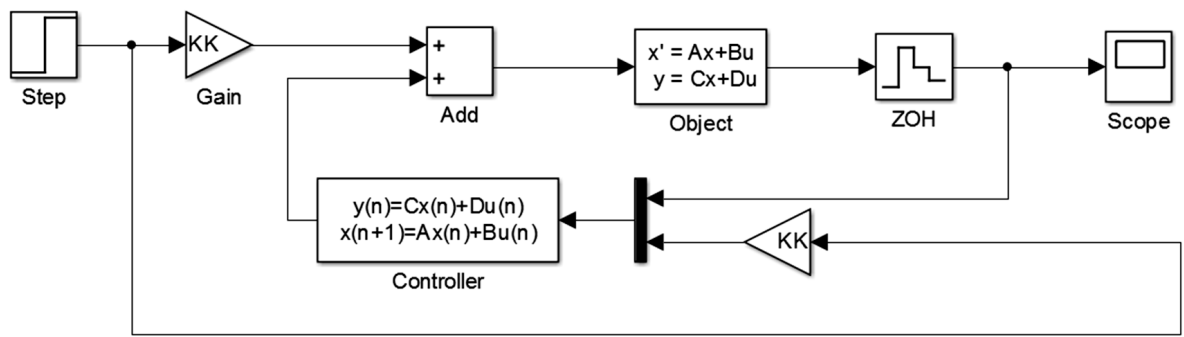

Figure 5: Simulink system.

The zero-order-hold is necessary to simulate a measurement device (e.g. thermometer) with various sampling times. We used the Tustin method (see e.g., Astrom 1990, [1, p. 212]) to discretize the compensator and then find the appropriate sampling frequency. It transforms the continuous system $S(A, B, C, D)$ into a discrete one for a given sampling time $h$ using the formulas

$$
\begin{aligned}
& A^{+}=\left(I+\frac{h}{2} A\right)\left(I-\frac{h}{2} A\right)^{-1} \\
& B^{+}=A^{-1}\left(A^{+}-I\right) B \\
& C^{+}=C \\
& D^{+}=D
\end{aligned}
$$

During the simulation we wanted to find optimal sampling time of the compensator for various sampling frequencies for temperature measurement. We used the performance indicator proposed by Bini and Buttazzo (2014) [3]:

$$
J(N)=\frac{1}{N} \int_{0}^{T}|\dot{u}(t)| d t
$$

During the simulations, we set $T=200$ [s]. For optimization, we used golden search with parabolic interpolation implemented in Matlab Optimization Toolbox. The optimization constraints were chosen as $1 \leqslant N \leqslant 10^{6}$. The results are gathered in Tab. 1 . 
Table 1: The results of optimization

\begin{tabular}{|c|c|c|}
\hline $\begin{array}{c}\text { Temperature sampling } \\
\text { frequency }[\mathrm{Hz}]\end{array}$ & $\begin{array}{c}\text { Optimal number } \\
\text { of samples } N_{\text {opt }}\end{array}$ & $\begin{array}{c}\text { Sampling time } \\
h=\frac{T}{N_{\text {opt }}}[\mathrm{s}]\end{array}$ \\
\hline 10 & 23700 & 0.0084 \\
\hline 1 & 23896 & 0.0083 \\
\hline 0.1 & 68957 & 0.0029 \\
\hline 0.03 & 84140 & 0.0024 \\
\hline 0.02 & 48284 & 0.0041 \\
\hline 0.01 & 69997 & 0.0028 \\
\hline
\end{tabular}

It can be seen that sampling time of the controller increases with increasing sampling frequency. This means that we have a buffer in the controller for doing necessary calculations. The accuracy of temperature measurements and controller performance are depicted in the Figs 6 and 7.

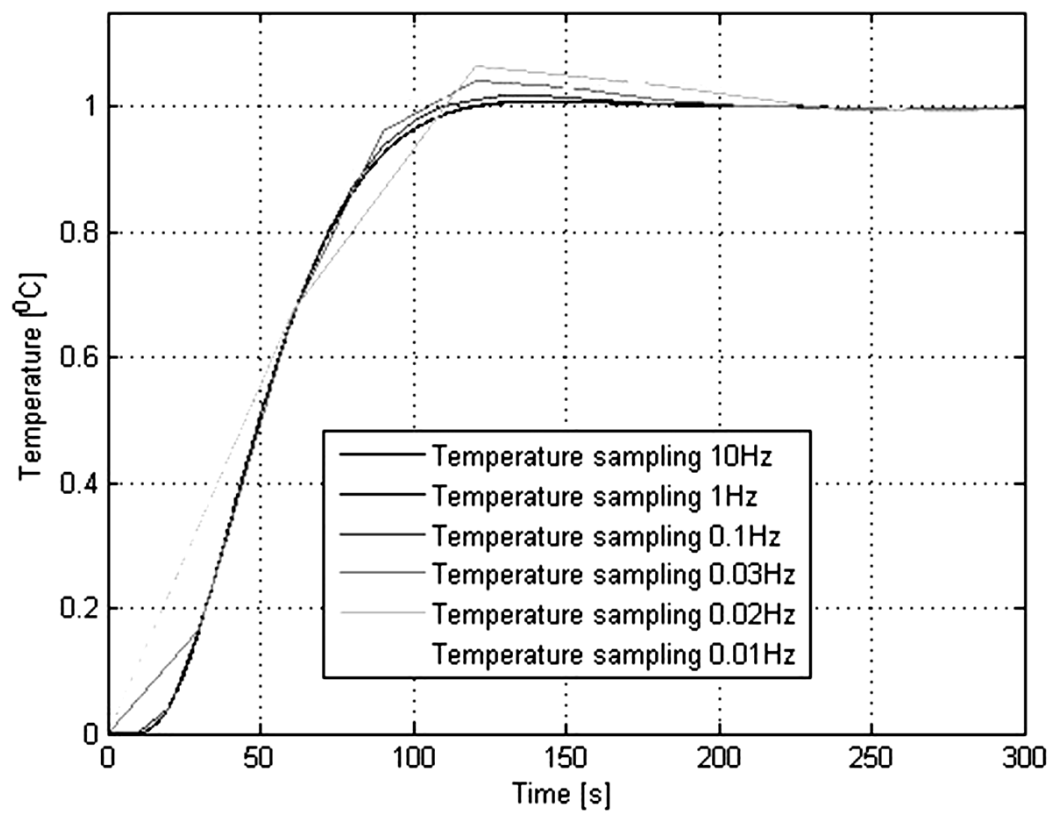

Figure 6: Temperature for various sampling frequencies. 


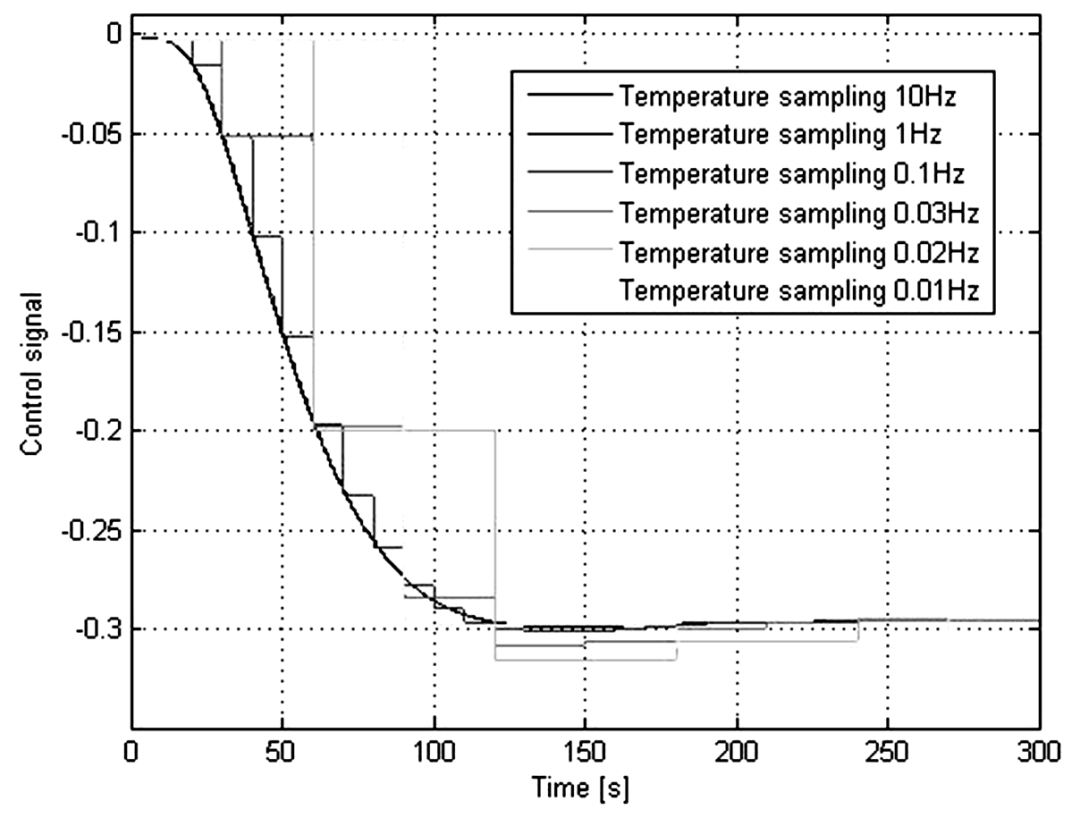

Figure 7: Control signal for various sampling frequencies.

\section{Comparison quality index}

For all numerical experiments the golden search with parabolic interpolation method has been chosen for tuning $N_{\text {opt }}$ parameter. Initial value for all experiments have value 1 .

The tests will be conducted for the following quality index:
1. $\frac{1}{N} \int_{0}^{T}|\dot{u}(t)| d t$,
2. $\frac{1}{N} \int_{0}^{T} u^{2} d t$
3. $\frac{1}{N} \int_{0}^{T}|u| d t$
4. $\frac{1}{N} \int_{0}^{T} t u d t$

I can bee seen, that all quality index give the same result for the same sampling frequencies (see Fig. 8, Fig. 9 and Fig. 10). But calculating are the faster for the quality index of form 1 (see Tab. 2) .

\section{Conclusion}

The main purpose of this work was to present possible way of approximating infinite dimensional systems with finite dimensional ones. The resulting system can then be discretized and implemented in digital controllers. The results were confirmed with simulation as we analyzed the process of thin rod heating. We found optimal sampling time 


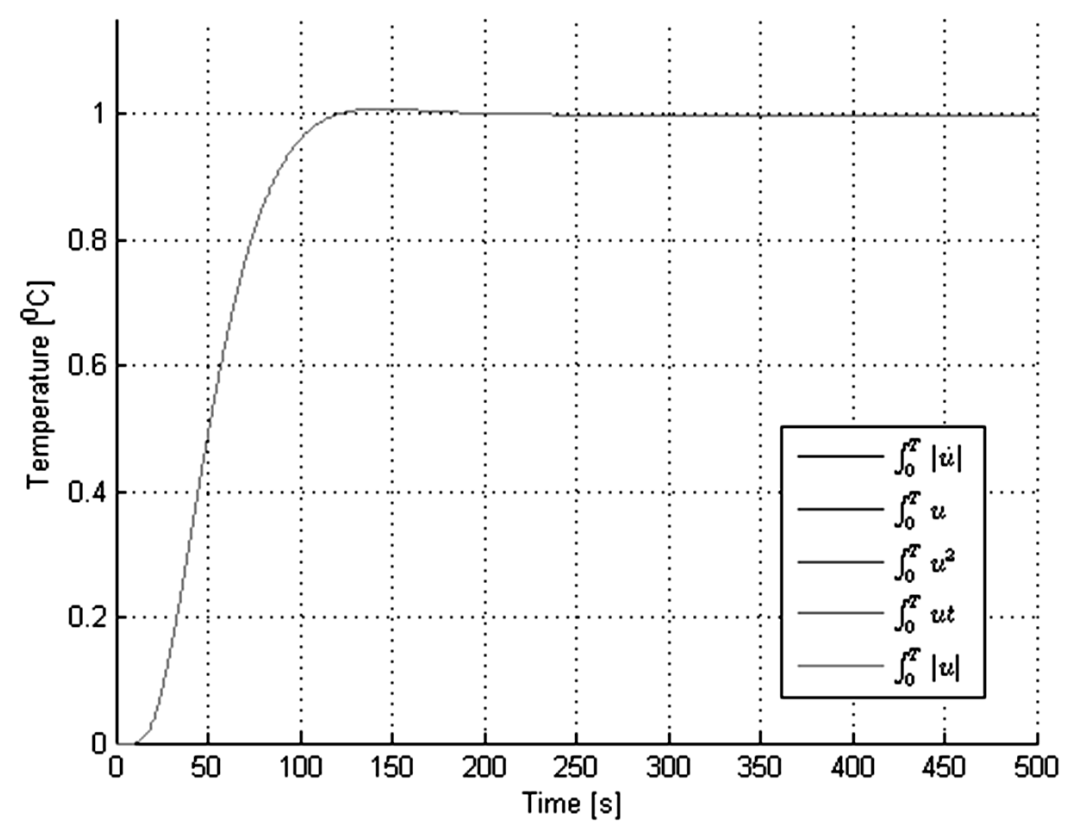

Figure 8: Temperature for various quality index with sampling frequencies $10 \mathrm{~Hz}$.

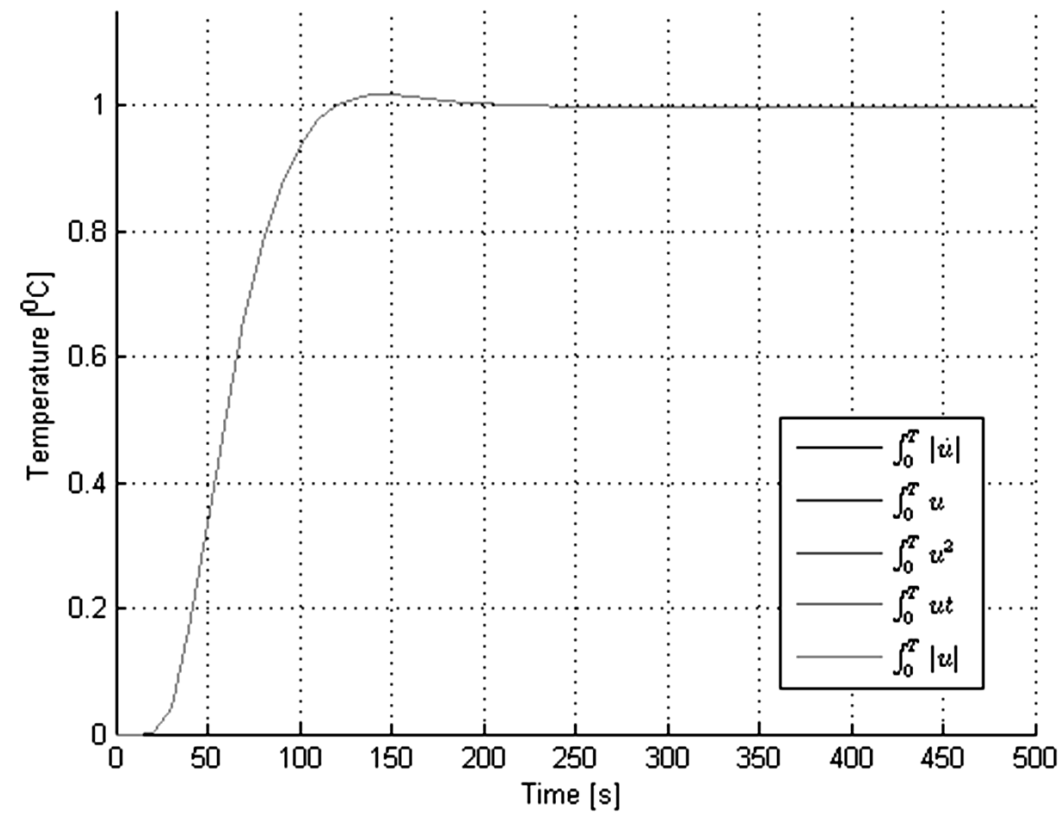

Figure 9: Temperature for various quality index with sampling frequencies $0.01 \mathrm{~Hz}$. 


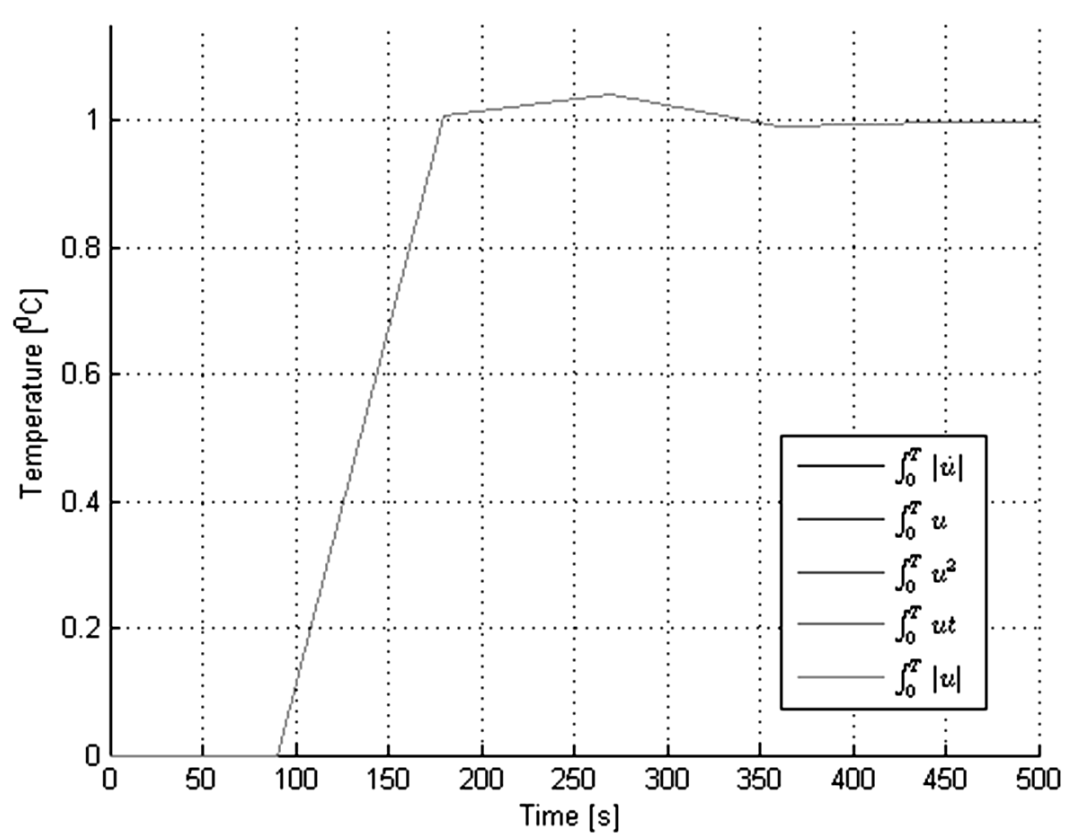

Figure 10: Temperature for various quality index with sampling frequencies $0.1 \mathrm{~Hz}$

for the compensator depending on various output sampling frequencies and comparison result for different quality index.

Nevertheless, the proposed algorithm is general and may be used for control of various systems. One of the possible way of applications may be non-integer order diffusion equation Gal and Warma (2016) [12], see also Evans (2007) [10]. However, it will require further analysis and research, as the methods for integer order systems cannot be directly applied to them.

\section{References}

[1] K.J. Astrom: Computer Controlled Systems. Theory and Design. Second Edition. Prentice-Hall, 1990.

[2] M.J. BALAS: The Galerkin method and feedback control of linear distributed parameter systems. J. Mathematical Analysis and Application, 91(2), (1983), 527546.

[3] E. Bini and G.M. Buttazzo: The optimal sampling pattern for linear control systems. IEEE Trans. On Automatic Control, 59(1), (2014), 78-90. 
Table 2: The results of optimization

\begin{tabular}{|c|c|c|c|}
\hline $\begin{array}{c}\text { Quality } \\
\text { index number }\end{array}$ & $\begin{array}{c}\text { Temperature sampling } \\
\text { frequency }[\mathrm{Hz}]\end{array}$ & Error & $\begin{array}{c}\text { Time } \\
\text { of calculation }[\mathrm{s}]\end{array}$ \\
\hline 1 & 10 & 0.0545 & 200 \\
& 1 & 0.0548 & 191 \\
& 0.1 & 0.0586 & 93 \\
& 0.03 & 0.0687 & 61 \\
& 0.02 & 0.0853 & 102 \\
\hline 2 & 0.01 & 0.0889 & 80 \\
\hline 3 & 10 & 0.0545 & 455 \\
& 1 & 0.0548 & 448 \\
& 0.1 & 0.0586 & 135 \\
& 0.03 & 0.0687 & 113 \\
& 0.02 & 0.0853 & 87 \\
& 0.01 & 0.0889 & 90 \\
\hline & 10 & 0.0545 & 466 \\
& 1 & 0.0548 & 454 \\
& 0.1 & 0.0586 & 158 \\
& 0.03 & 0.0687 & 119 \\
& 0.02 & 0.08537 & 87 \\
& 0.01 & 0.08897 & 90 \\
\hline 4 & 10 & 0.0545 & 441 \\
& 1 & 0.0548 & 432 \\
& 0.1 & 0.0586 & 173 \\
& 0.03 & 0.0687 & 116 \\
& 0.02 & 0.0853 & 102 \\
& 0.01 & 0.0889 & 101 \\
\hline
\end{tabular}

[4] R.F. CURTAIN: Finite dimensional compensators for parabolic distributed systems with unbounded control and observation. SIAM J. Control and Optimization, 22(2), (1984), 255-276.

[5] R.F. Curtain and D. SAlomon: Finite dimensional compensators for infinite dimensional systems with unbounded input operators. SIAM J. Control and Optimization, 24(4), (1986), 797-816.

[6] R.F. CURTAIN: Linear-quadratic control problem with fixed endpoints in infinite dimensions, J. of Optimization Theory and its Applications, 44(1), (1984), 55-74.

[7] R.F. Curtain and A.J. Pritchard: Infinite-Dimensional Linear Systems Theory. Springer, Berlin, 1978. 
[8] R.F. CURTAIN and H. ZWART: An Introduction to Infinite-Dimensional Linear Systems Theory. Springer, New York, NY, 1995.

[9] Z. EMIRSAJŁOW: Feedback control in LQCP with a terminal inequality constraint. J. of Optimization Theory and Applications, 62(3), (1989), 387-403.

[10] K.P. EvAnS and N. JACOB: Feller semigroups obtained by variable order subordination. Revista Matematica Complutense, 20(2), (2007), 293-307.

[11] H.O. FATTORINi: On complete controllability of linear systems. J. Differential Equations, 3(3), (1967), 391-402.

[12] C. GAL and M. WARMA: Elliptic and parabolic equations with fractional diffusion and dynamic boundary conditions. Evolution Equations and Control Theory, 5(1), (2016), 61-103.

[13] J.S. GiBSON: An analysis of optimal modal regulation: convergence and stability. SIAM J. Control and Optimization, 19(5), (1981), 686-707.

[14] E.W. KAmEn, P.P. Khargonekar and A. TAnnEnbaum: Stabilization of timedelay systems using finite-dimensional compensators. IEEE Trans. on Automatic Control, 30(1), (1985), 75-78.

[15] T. Kato: Perturbation Theory for Linear Operators. Springer, Berlin, 1966.

[16] T. Kobayashi: Discrete-time observers and parameter determination for distributed parameter systems with discrete-time input-output data. SIAM J. on Control and Optimization, 21(3), (1983), 331-351.

[17] W. Mitkowski: Stabilization of linear distributed systems. 3rd Symp. IFAC, Control of Distributed Parameter Systems, Toulouse, France, (1982), p. IV.10-IV.13.

[18] W. MitKOWSKI: Feedback stabilization of second order evolution equations with damping by discrete-time input-output data. IMACS-IFAC Symp. on Modelling and Simulation for Control of Lumped and Distributed Parameter Systems, Lille, France, (1986), 355-358.

[19] W. Mitkowski: Stabilizacja liniowych układów nieskończenie wymiarowych za pomocą dynamicznego sprzężenia zwrotnego (Stabilization of infinite-dimensional linear systems by dynamic feedback). Archiwum Automatyki i Telemechaniki, 33(4), (1988), 515-528, (in Polish).

[20] W. MitKowsKi: Stabilizacja systemów dynamicznych (Stabilization of Dynamic Systems). WNT, Warszawa, 1991.

[21] W. Mitkowski and K. Oprzędkiewicz A sample time assign for a discrete interval parabolic system with the two-dimensionalmuncertain parameter space. Systems Science, 30(1), (2004), 43-50. 
[22] A. ObRaczKa and W. Mitkowski: The comparison of parameter identification methods for fractional partial differential equation. Solid State Phenomena, 21 (2014), 265-270.

[23] K. OPRZEDKIEWICZ: The interval parabolic system. Archives of Control Sciences, 13(4), (2003), 415-430.

[24] K. OprzęDKIEwicz, E. Gawin and W. Mitkowski: Modeling of heat distribution with the use of non-integer order, state space model. Int. J. of Applied Mathematics and Computer Science, 26(4), (2016), 749-756.

[25] A. PAZY Semigroups of Linear Operators and Applications to Partial Differential Equations. Springer, New York, 1983.

[26] K.M. PRZYŁUSKI: On an infinite dimensional linear-quadratic problem with fixed endpoints: The continuity question. Int. J. of Applied Mathematics and Computer Science, 24(4), 723-733.

[27] Y. SAKAWA: Feedback stabilization of linear diffusion systems. SIAM J. Control and Optimization, 21(5), (1983), 667-676.

[28] Y. SAKAWA: Feedback control of second order evolution equations with damping. SIAM J. Control and Optimization, 22(3), (1984), 343-361.

[29] Y. SAKAWA: Feedback control of second-order evolution equations with unbounded observation. Int. J. Control, 41(3), (1985), 717-731.

[30] J.M. SchUmACher: Dynamic Feedback in Finite- and Infinite -dimensional Linear Systems. Ph. D. dissertation, Dep. Math., Vrije Universiteit, Amsterdam, The Netherlands, 1981.

[31] J.M. SCHUMACHER: A direct approach to compensator design for distributed parameter systems. SIAM J. Control and Optimization, 21(6), (1983), 823-836.

[32] D. Sierociuk, T. Skovranek, M. Macias, I. Podlubny, I. Petras, A. DZIELINSKI and P. ZIUBINSKI: Diffusion process modeling by using fractionalorder models. Applied Mathematics and Computation, 257(1), (2015), 2-11.

[33] M. SLEMROD: A note on complete controllability and stabilizability for linear control systems in Hilbert space. SIAM J. Control, 12(3), (1974).

[34] R. TRIgGiani: On the stabilization problem in Banach space. J. of Mathematical Analysis and Applications, 52(3), (1975), 383-403.

[35] P.K.C. WANG: Model feedback stabilization of linear distributed system. IEEE Trans. on Automatic Control, 17(4), (1972), 552-553. 